\title{
Implikaturen im Bereich lexikalisch induzierter Präsuppositionen
}

auch wenn-Konstruktionen können unter bestimmten Bedingungen konzessiv interpretiert werden. In Wörterbüchern und Grammatiken wird deshalb wenn neben einer konditionalen auch eine konzessive Bedeutung zugeschrieben. Es soll gezeigt werden, daß dies für auch wenn-Konstruktionen nicht erforderlich ist, daß bei diesen vielmehr die konzessive Interpretation aus einer von auch induzierten konditionalen Präsupposition und einer mit Konditionalität verknüpften Implikatur abgeleitet werden kann, die Geis/Zwicky auf das Wirken des Prinzips der "conditional perfection" zurückführen.

Diese Implikatur ist eine "konzeptuelle Implikatur". Konzeptuelle Implikaturen, die von Präsuppositionen induziert werden, sind nicht in der Weise aufhebbar, wie es Implikaturen sind, die von Aspekten der Bedeutung induziert werden. Sie können als aktuelle Inferenzen nur durch die Bedeutung verhindert werden, für die die Präsupposition induziert wird, durch die sie selbst induziert werden. Wenn die Aufhebbarkeit einer Implikatur derart beschränkt ist, müssen die von Grice (1975) formulierten und in der Literatur (siehe Levinson 1983:113) verfeinerten Kriterien für die Konversationalität von Implikaturen noch weiter verfeinert werden.

Der Inhalt einer von der Äußerung eines sprachlichen Ausdrucks $a_{i}$ auszulösenden (pragmatischen) Implikatur - ein pragmatisches Implicatum, im folgenden kurz: "eine Implikatur" genannt - ist etwas, von dem der Hörer der Äußerung von $a_{i}$ annehmen kann, daß es bei der Verwendung von $a_{i}$ interpretiert werden kann, das aber nicht als konventionelle Eigenschaft von $\mathrm{a}_{\mathrm{i}}$ anzusehen ist.

In der Literatur, die sich mit dem Phänomen der Implikaturen befaßt, wird im allgemeinen davon ausgegangen, daß im Gegensatz zu konventionellen - semantischen - Implicata Implikaturen durch den Sprecher als nicht intendiert charakterisiert werden können, ohne daß das, was der Sprecher ausdrückt, als inkonsistent erscheint. Siehe Grice (1975:57): "A generalized conversational implicature can be canceled in a particular case." Vgl. (1) gegenüber (2)(a) und (b):

A.: Wenn Messe ist, schreibt die Straßenbahngesellschaft schwarze Zahlen.

B.: Und sonst nicht?

A.: Doch, aber zur Messe ganz besonders.

(2)(a) A.: Ich bin krank.

B.: Du bist krank?

A.: ? Ich bin ganz gesund.

(2)(b) A.: Mein Hund ist überfahren worden.

B.: Was? Du hast einen Hund?

A.: ?Ich habe keinen Hund.

Die in (1) von B. abgeleitete Implikatur kann mit 'wenn keine Messe ist, schreibt die StraBenbahngesellschaft keine schwarzen Zahlen' umschrieben werden. Dieses Phänomen der Interpretation einer Konditionalbeziehung zwischen dem Negat des Antezedens und dem Negat des Konsequens einer Konditionalverknüpfung wird als Prinzip der "conditional perfection" - CP - bezeichnet (siehe Geis/Zwicky 1971). 
Im folgenden soll gezeigt werden, daß das, was das Prinzip der conditional perfection abzuleiten gestattet, nicht nur zu Implikaturen im Bereich der Äußerungsbedeutung sprachlicher Ausdrücke führt, sondern auch spezifische Diskurspräsuppositionen abzuleiten erlaubt, "präsuppositionale Implikaturen", wie ich sie nennen möchte. Daraus wiederum leite ich die Annahme ab, daß es konzeptuelle Implikaturen gibt, deren Tilgbarkeit davon abhängt, ob sie für den präsuppositionalen oder den Bedeutungsaspekt sprachlicher Ausdrücke abzuleiten sind.

$\mathrm{Zu}$ diesem Zweck betrachte ich hier speziell Satzverknüpfungen mit der deutschen Konjunktion wenn, der ein auch vorangestellt ist.

Konditionale Satzverknüpfungen mit auch können unter bestimmten Bedingungen konzessiv interpretiert werden; vgl. Ist es auch Wahnsinn, hat es doch Methode; wenn es auch Wahnsinn ist, (so) hat es doch Methode; auch wenn es Wahnsinn ist, (so) hat es doch Methode. In Wörterbüchern und Grammatiken aber auch in speziellen Untersuchungen zu konzessiven Konjunktionen wird deshalb wenn neben einer konditionalen auch eine konzessive Bedeutung zugeschrieben, oder auch wenn und wenn auch werden dort als komplexe Konjunktionen behandelt.

Wenn bei der Untersuchung der Verwendungsbedingungen lexikalischer Einheiten und dabei speziell von Konjunktionen in größerem Maße als bisher Implikaturen der ihnen gebührende Platz eingeräumt wird, ist es in vielen Fällen nicht erforderlich, von einer derartigen Mehrdeutigkeit lexikalischer Einheiten auszugehen. Es wird gleichzeitig auch erklärbar, warum bestimmte lexikalische Einheiten die unterstellten Mehrdeutigkeiten aufweisen bzw. die illustrierten Kombinationen mit Partikeln mit ganz bestimmten inhaltlichen Effekten eingehen, andere dagegen nicht.

Dies soll im folgenden anhand von auch wenn-Konstruktionen gezeigt werden. Es soll demonstriert werden, daß sich die Interpretation von auch wenn-Konstruktionen aus folgenden Faktoren ableiten läßt:

1. aus der auf die materiale Implikation zurückgeführten Konditionalinterpretation von wenn

2. aus den Gebrauchsbedingungen von auch, und zwar speziell einer von auch induzierten Präsupposition

3. aus einer mit dem Begriff der Konditionalität verbundenen Implikatur

4. aus einer an die Spezifik der Bedeutungen der durch wenn verknüpften Sätze gebundenen Erfahrung von möglichen Zusammenhängen der von den Sätzen bezeichneten Sachverhalte und

5. aus der Interpretation des vom wenn-Satz bezeichneten Sachverhalts als Tatsache (faktisch). 


\section{Konzessivität von Satzverknüpfungen}

Um zeigen zu können, wie sich die konzessive Interpretation von wenn-Konstruktionen ergibt, muß der Begriff der Konzessivität inhaltlich einigermaßen kontrollierbar gefaßt werden.

Typische konzessive Satzverknüpfungen sind solche, die mit den Konjunktionen obwohl, obschon oder wenngleich gebildet werden. $\mathrm{Vgl}$.:

(3) [A. gibt sich ungehalten. B.:]

Obwohl/obschon/wenngleich es dir lästig ist, mußt du mir jetzt zuhören.

Als Quintessenz aus zahlreichen Arbeiten zu konzessiven Satzverknüpfungen leite ich ab, $\mathrm{da} ß$ eine konzessive Satzverknüpfung mindestens folgendem Schema genügen muß:

Schema der Interpretation konzessiver Satzverknüpfungen:

(Diskurs-)Präsupposition:p --> q

Bedeutung: $p$ \& q

(Eine ähnliche Repräsentation findet sich bei König 1986:234.)

Dabei ist $p$ eine Variable über die von den untergeordneten Sätzen ausgedrückten Propositionen, $q$ eine Variable über die von den übergeordneten Sätzen ("Bezugssätzen") ausgedrückten Propositionen, \& ein Zeichen für die logische Konjunktion, --> ein Zeichen für die materiale Implikation und ein Zeichen für die aussagenlogische Negation.

Unter der "Bedeutung" eines sprachlichen Ausdrucks verstehe ich hier das, was dieser Ausdruck zu den Wahrheitsbedingungen von (einfachen und komplexen) Deklarativsätzen beitragen kann.

Unter einer mit einem sprachlichen Ausdruck verknüpften "Präsupposition" verstehe ich hier den Inhalt einer vor der Verwendung dieses Ausdrucks getroffenen oder zu treffenden Vorannahme, die einen spezifischen konzeptuellen Hintergrund für die Bedeutung des Ausdrucks bildet. (Ich verstehe "Präsupposition" im Sinne des Begriffs der "discourse presupposition", wie ihn Givón 1978 geprägt hat.)

Ich setze voraus, daß Präsupposition und Bedeutung zusammen in einem logischem Ausdruck vorkommen. Als Funktor, der sie verknüpft, nehme ich einen pragmatischen Funktor - PRIOR - an, der einen Anspruch auf die Wahrheit der "Präsupposition" (eigentlich: des Präsupponierten) zeitlich der epistemischen Bewertung (Urteil, Frage usw.) dessen, was hier "Bedeutung" genannt wird, vorordnet. $p$ und $q$ und die Beziehung, die zwischen ihnen durch die Bedeutung des Konnektivs etabliert wird, sind semantischen Typen zugeordnet, die syntaktischen Kategorien zugeordnet sind. Für PRIOR und die Funktoren im Bereich der Präsuppositionen gilt dies nicht. Sie sind nur konzeptuellen Typen zugeordnet. (Die semantisch typisierten Einheiten sind zweifach typisiert: semantisch und konzeptuell.) Die epistemischen Bewertungen modalisieren die Propositionen. Sie weisen sie z.B. als Inhalt eines Urteils, einer Frage, einer Aufforderung aus. Nur wenn eine Proposition als Urteilsinhalt modalisiert ist, kann das Urteil, daß die Proposition wahr bzw. falsch ist, gefällt werden. Ich nehme an, daß präsuppositionale Propositionen immer als Urteilsinhalte moda- 
lisiert sind. Durch die logische Schlußregel der Einführung der logischen Konjunktion (EK) können Urteilsinhalte durch logische Konjunktion miteinander verbunden werden.

In logischer Konjunktion ergibt die "Konzessivbedeutung" mit der "Konzessivpräsupposition" (siehe $(\mathrm{K})$ ) bei allen Verteilungen der Wahrheitswerte von $\mathrm{p}$ und q den Wert falsch, also eine Kontradiktion. Das Wesen der Konzessivität ist ein Widerspruch zwischen Präsupposition und Bedeutung, insbesondere Negation der präsuppositionalen Folge q durch die Bedeutungskomponente q. Den Widerspruch als konstitutives Merkmal konzessiver Satzverknüpfungen unterstellen auch in der Regel die einzelsprachlichen Bedeutungswörterbücher.

Die Präsupposition konzessiver Konstruktionen - im folgenden: "Konzessivpräsupposition" - ist also eine Konditionalbeziehung, die auf die Wahrheitsfunktion der materialen Implikation zurückzuführen (wenn auch nicht mit dieser zu identifizieren) ist. Diese hat die Wahrheitswertcharakteristik <w,w; f,w; f,f $>$. (In den in spitzen Klammern aufgeführten Wertepaaren steht jeweils der linke Wert für den Wert des Antezedens - der Bedingung -, der rechte für den des Konsequens - der Folge - der Konditionalbeziehung.) D.h. die in ihrer Bedeutung auf diese Wahrheitsfunktion zurückzuführenden Satzverknüpfungen gelten nur dann als falsch, wenn der Satz, der die Bedingung in der Konditionalbeziehung ausdrückt, wahr ist und der Satz, der die Folge in der Konditionalbeziehung ausdrückt, falsch ist. Bei den restlichen drei möglichen Verteilungen der Wahrheitswerte der zwei verknüpften Sätze gelten die entsprechenden Satzverknüpfungen als wahr. Im Unterschied zur Bedeutung von Konditionalkonstruktionen besteht aber bei konzessiv zu interpretierenden Satzverknüpfungen diese Konditionalbeziehung nicht zwischen $\mathrm{p}$ und $\mathrm{q}$, sondern zwischen $\mathrm{p}$ und $\tilde{q}$.

Gegen die Zurückführung der Konditionalbeziehung auf eine Wahrheitsfunktion und dabei speziell auf die materiale Implikation der Aussagenlogik äußern viele Linguisten und Logiker Vorbehalte. Derzeit steht jedoch meines Wissens keine Logik zur Verfügung, in der der für Konzessivinterpretationen von Satzverknüpfungen relevante Widerspruch zwischen Präsupponiertem und Bedeutungen der Satzverknüpfungen formal ableitbar wäre. Führt man die Konzessivinterpretationen von Satzverknüpfungen jedoch auf die materiale Implikation zurück, so kann man, auf der Grundlage der Annahme einer Ableitung der logischen Konjunktion von Präsupponiertem und Bedeutung der Satzverknüpfungen, diesen Widerspruch recht gut formal veranschaulichen.

Als nichtwahrheitsfunktional sieht z.B. Wessel (1984:296) den Konditionaloperator an. Er weist Satzverknüpfungen bei den Werteverteilungen $<w$,w; f,w; f,f $>$ keinen der beiden Werte w bzw. f zu. Er läßt den Wert der Satzverknüpfung unbestimmt, was er durch "?" notiert. Bei der Werteverteilung w,f weist er den Konditionalkonstruktionen den Wert zu, den auch die materiale Implikation bei dieser Verteilung der Wahrheitswerte der verknüpften Sätze ergibt, nämlich den Wert $\mathrm{f}$.

Dies soll folgende Wahrheitswertetafel ausdrücken: 


\begin{tabular}{l|l||c} 
A & B & A $-->$ B \\
\hline w & w & $?$ \\
w & f & f \\
f & w & $?$ \\
f & f & $?$
\end{tabular}

Wenn nun diese Interpretation von --> auf $(\mathrm{K})$ angewandt wird, ergibt sich bei logischer Konjunktion der Präsupposition p --> $\sim q$ und der Bedeutung p \& q aus (K) - anders als wenn der Pfeil -- $>$ als Zeichen der materialen Implikation interpretiert wird - keine Kontradiktion im Sinne der klassischen zweiwertigen Logik, als die der Widerspruch, der in Konzessivinterpretationen relevant wird, gedeutet werden kann. Eine Kontradiktion würde ja nur dann vorliegen, wenn die logische Konjunktion von Präsupposition und Bedeutung bei allen Werteverteilungen den Wert $\mathrm{f}$ annimmt. Die für Konzessivkonstruktionen typische Kontradiktion kann sich bei der von Wessel vorgeschlagenen Interpretation des Konditionaloperators auf der Grundlage des Kontradiktionsbegriffs der klassischen zweiwertigen Logik nicht ergeben, weil die logische Konjunktion im Rahmen dieser Logik nur für die Werte $w$ und $f$ definiert ist. In diesem Rahmen nimmt deshalb die logische Konjunktion als Verknüpfung von Konzessivpräsupposition und Konzessivbedeutung den Wert $f$ nur dann an, wenn sowohl $\mathrm{p}$ als auch $\mathrm{q}$ den Wert $w$ annimmt. In diesem Fall ist die Konzessivbedeutung wahr und die Konzessivpräsupposition falsch. (Letztere ist ja falsch, wenn $p$ wahr und $q$ falsch, also q wahr ist.) Für die anderen Werteverteilungen, für die sich bei der Konzessivpräsupposition der Wert "?" ergibt, ist die Konzessivbedeutung zwar falsch, aber es kann bei der Verknüpfung von Konzessivpräsupposition und Konzessivbedeutung durch logische Konjunktion aufgrund der Unbestimmtheit des Wahrheitswertes der Konzessivpräsupposition - d.h. aufgrund des Wertes "?" - im Rahmen einer zweiwertigen Logik nicht entschieden werden, welchen Funktionswert diese logische Konjunktion ergibt: "?" oder $f$.

Ein Ausweg wäre es, den Begriff der Kontradiktion weiter zu fassen und ihn auf die Verknüpfung von Konzessivpräsupposition und -Bedeutung auf der Grundlage der von Wessel vorgeschlagenen nichtwahrheitsfunktionalen Interpretation von --> zuzuschneiden. Dann müßte man vorsehen, 1 . daß neben den Werten w und f noch ein dritter Wert "?" vorliegen kann, 2. daß die logische Konjunktion keine zwei-, sondern eine dreiwertige Aussagenfunktion ist und 3. daß der Funktionswert der logischen Konjunktion von Konzessivpräsupposition und Konzessivbedeutung immer $f$ sein soll, wenn eines der Argumente dieser logischen Konjunktion der Wert $f$ ist. Letzteres ließe sich damit rechtfertigen, daß die logische Konjunktion auch in der zweiwertigen Logik immer den Wert $f$ annimmt, wenn mindestens eines ihrer beiden Argumente $f$ ist. Offen bliebe dabei allerdings die Frage, welchen Wert die logische Konjunktion annimmt, wenn eines ihrer beiden Argumente w ist und das andere "?" oder wenn beide Argumente "?" sind.

Angesichts dieser offenen Fragen halte ich an der Zurückführung der Konditionalbeziehung auf die Wahrheitsfunktion der materialen Implikation fest. 


\section{Konzessivität von wenn-Konstruktionen}

\section{1. sogar wenn- und selbst wenn-Konstruktionen}

In der Literatur zur konzessiven Interpretation von Konstruktionen, die mittels einer konditional zu interpretierenden Konjunktion gebildet werden, werden vorwiegend engl. even if und franz. même si behandelt. Die konzessive Interpretation wird für diese Wortverbindungen darauf zurückgeführt, daß der vom unmittelbar auf sie folgenden Satz bezeichnete Sachverhalt [[p]] einen Extremwert auf einer Skala möglicher Bedingungen für den vom übergeordneten Satz der Satzverknüpfung bezeichneten Sachverhalt [[q]] darstelle. Dies werde durch die Gradpartikel even bzw. même ausgedrückt. Ein Extremwert sei [[p]] insofern, als unerwartet sei, daß die vom Konditionalsatz (d.h. vom if- bzw. vom si-Satz) bezeichnete Bedingung die vom übergeordneten Satz bezeichnete Folge zeitige. Normalerweise folge aus dem vom if- bzw. vom si-Satz bezeichneten Sachverhalt das Gegenteil des vom übergeordneten Satz bezeichneten Sachverhalts. Nach König (1986:232) präsupponiert even "that the value given in the focus (des Satzes, von dem even Konstituente ist - R.P.) is the least likely and therefore most suprising of all values under consideration in a given context." (Ähnlich beschreiben die Bedeutung von even Karttunen/Peters 1979:24ff.)

Man kann es auch so sehen: engl. even, französisch même und deutsch selbst oder sogar induzieren, wenn eine Konditionalverknüpfung mit der Bedeutung $\mathrm{p} \mathrm{-->} \mathrm{q} \mathrm{in} \mathrm{ihrem} \mathrm{Skopus}$ liegt, die Präsupposition ( $p-->$ q). Diese ergibt in logischer Konjunktion mit der Bedeutung der von der Konditionalkonjunktion gestifteten Satzverknüpfung p --> q einen Widerspruch (immer den Wahrheitswert falsch). Das heißt, solche Konstruktionen weisen ein für konzessive Konstruktionen typisches Merkmal auf.

\section{2. auch wenn-Konstruktionen}

Diese Charakterisierung der Leistung von sogar und selbst läßt sich jedoch nicht auf deutsch auch übertragen. Vgl. (4)(a) und (4)(b):

(4)(a) Auch ich war ein Jüngling im lockigen Haar.

(b) Sogar ich war ein Jüngling im lockigen Haar.

(4)(a) besagt im Unterschied zu (4)(b) nur, daß neben dem Sprecher der Satzverknüpfung auch noch andere Personen Jünglinge im lockigen Haar waren, nicht jedoch, daß zu erwarten war, daß der Sprecher kein Jüngling im lockigen Haar war.

Das heißt, für die im Deutschen vor der Konditionalkonjunktion in konzessiv zu interpretierenden Konstruktionen zu verwendende Partikel auch ist die Annahme, daß der von dem sie enthaltenden Satz bezeichnete Sachverhalt [[p]] einen Extremwert auf einer Skala darstellt, unplausibel. Letztlich ist es dieses Fehlen der Extremwertvorstellung, das die Gebrauchsbedingungen der fokussierenden Partikel auch von denen der fokussierenden Partikeln sogar oder selbst (den direkten Entsprechungen von engl. even und franz. même) unterscheidet. Vgl. die intuitiven Unterschiede zwischen den Beispielen in (5) und (6): 
(5) [A. gibt sich ungehalten. B.:]

(a) Auch wenn es dir lästig ist, mußt du mir jetzt zuhören.

(b) Auch wenn es dir lästig ist, du mußt mir jetzt zuhören.

(c) Auch wenn es dir lästig ist, (so) mußt du mir doch jetzt zuhören.

(6) [A. gibt sich ungehalten. B.:]

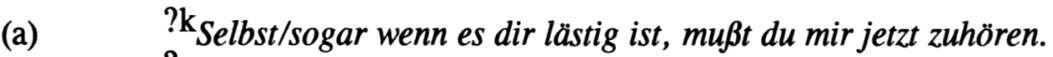

(b) ?Selbst/sogar wenn es dir lästig ist, du mußt mir jetzt zuhören.

(c) ?Selbst/sogar wenn es dir lästig ist, (so) mußt du mir doch jetzt zuhören.

(Der Index k hinter dem indizierten Fragezeichen soll den folgenden Text als im gegebenen Kontext unangemessen kennzeichnen.)

auch weist vielmehr Gebrauchsbedingungen auf, die aus seiner historischen Verwandtschaft mit und herrühren. Seine Bedeutung kann auf die Wahrheitsfunktion der logischen Konjunktion zurückgeführt (wenn auch nicht mit dieser identifiziert) werden. Es induziert eine Präsupposition, mit der die Bedeutung des Satzes, von dem auch Konstituente ist, durch auch in logische Konjunktion gebracht wird.

(AWK) Schema der Interpretation von auch wenn-Konstruktionen:

(a) Präsupposition (von auch induziert): $\mathrm{r}-->\mathrm{q}$

(b) Bedeutung: 1. ( $\mathrm{r}-->\mathrm{q}) \&(\mathrm{p}-->\mathrm{q})$ wobei $\sim(r=p)$

ausgedrückt durch:

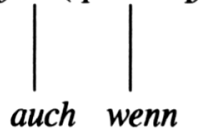

2.2.1. Der Beitrag lexikalisch induzierter Präsuppositionen zur Konzessivität von auch wenn-Konstruktionen

Wie ist dann jedoch die (K) erfüllende konzessive Interpretation von Satzverknüpfungen wie (5)(a) zu erklären?

Dem Augenschein nach haben konzessive Satzverknüpfungen gar nichts mit auch wennKonstruktionen, wie sie durch (AWK) schematisch beschrieben werden, gemein. Auf der Suche nach den Gemeinsamkeiten läßt sich jedoch ein Bindeglied ermitteln, das zur konzessiven Interpretation von auch wenn-Kon struktionen führt.

Dieses Bindeglied besteht in folgendem: Wenn die Partikel auch die Präsupposition $\mathrm{r}-->\mathrm{q}$ induziert und (wie in (AWK) angegeben) $\sim(r=p)$ gilt, dann läßt sich nach dem Prinzip der Äquivalenz von äußerer - den Operator einschließender - Negation und innerer nur den Operanden betreffender - Negation $r=\sim p$ ableiten und aus diesem wiederum - da ja r --> q präsupponiert ist - die Präsupposition p --> q. Die Ableitbarkeit ist garantiert, wenn die Identifikation zweier Propositionen - hier $r$ und $p$ - als symmetrische Relation definiert wird und ihr als aussagenlogisches Minimum die logische Äquivalenz zugrunde 
gelegt wird, die ja die Wahrheitswertcharakteristik <w,w; f,f $>$ hat. Diese besagt, daß die durch die Aussagenfunktion der Äquivalenz definierte Satzverknüpfung genau dann wahr ist, wenn sowohl $p$ als auch $r$ entweder der Wert wahr - w - oder der Wert falsch - f zukommt. Es läßt sich dann zeigen, daß $\sim(r=p)$ äquivalent mit $r=\sim p$ ist:

\begin{tabular}{l|l||l|l|l}
$\mathrm{r}$ & $\mathrm{p}$ & $\mathrm{r}=\mathrm{p}$ & $\sim(\mathrm{r}=\mathrm{p})$ & $\mathrm{r}=\mathrm{p}^{\mathrm{p}}$ \\
\hline $\mathrm{w}$ & $\mathrm{w}$ & $\mathrm{w}$ & $\mathrm{f}$ & $\mathrm{f}$ \\
$\mathrm{w}$ & $\mathrm{f}$ & $\mathrm{f}$ & $\mathrm{w}$ & $\mathrm{w}$ \\
$\mathrm{f}$ & $\mathrm{w}$ & $\mathrm{f}$ & $\mathrm{w}$ & $\mathrm{w}$ \\
$\mathrm{f}$ & $\mathrm{f}$ & $\mathrm{w}$ & $\mathrm{f}$ & $\mathrm{f}$
\end{tabular}

(AWK) kann dann zu (AWK') abgewandelt werden:

(AWK') Schema der Interpretation von auch wenn-Konstruktionen:

(a) Präsupposition (von auch induziert): $\mathrm{p}-->\mathrm{q}$

(b) Bedeutung: ( $(\mathrm{p}->\mathrm{q}) \&(\mathrm{p}->\mathrm{q})$ ausgedrückt durch:
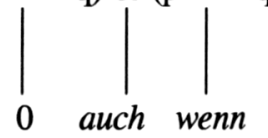

Die Bedeutung in (AWK') zeigt, daß auch wenn-Konstruktionen Irrelevanzkonditionale (König/Eisenberg 1984) sind: q folgt sowohl aus p als auch aus p, d.h. Bedingungen sind für die Wahrheit von q "irrelevant".

2.2.2. Das Zünglein an der Waage bei der Konzessivität von auch wenn-Konstruktionen: die präsuppositionale Konditionalimplikatur

Das Schema (AWK') weist nun jedoch nicht die für Konzessivität charakteristische Präsupposition p -- > q auf. Es stellt sich die Frage, ob diese direkt auf die Gebrauchsbedingungen der Konjunktion wenn zurückgeführt werden kann. Wohl kaum, sind doch wenn-Satzverknüpfungen ohne dem wenn voraufgehendes oder folgendes auch eher befremdlich. Vgl. gegenüber (5)(a) bis (c) oder Wenn es dir auch lästig ist, du mußt mir jetzt zuhören. oder Wenn es dir auch lästig ist, (so) mußt du mir doch jetzt zuhören. im Kontext von (5) die Konstruktionen (7)(a) bis (c):

(7) [A. gibt sich ungehalten. B.:]

(a) ?Wenn es dir lästig ist, mußt du mir jetzt zuhören.

(b) ?Wenn es dir lästig ist, du mußt mir jetzt zuhören.

(c) ?Wenn es dir lästig ist, (so) mußt du mir doch jetzt zuhören.

Woran liegt es, daß die Beispiele (7) befremdlich wirken? 
Das, was sie ausdrücken, steht einer Erfahrung entgegen, die der, der sie liest, normalerweise mit Sachverhalten der Art verbindet, die jeweils von den Bedeutungen der Teilsätze der Satzverknüpfungen identifiziert wird. Gemäß dieser Erfahrung folgt aus dem vom wenn-Satz bezeichneten Sachverhalt eben nicht der vom übergeordneten Satz bezeichnete Sachverhalt, sondern eher sein Gegenteil. Der Inhalt der Erfahrung ist eben die Präsupposition $\mathrm{p}-->\sim$ q, die von konzessiven Satzverknüpfungen induziert wird.

Die Präsupposition p --> q wird in (AWK') nicht angeführt. Wodurch ist sie zwar mit (AWK'), nicht aber generell mit Konditionalkonstruktionen ohne auch (siehe die Sätze (7)) verträglich?

Die Antwort auf diese Frage findet sich, wenn man das eingangs genannte und illustrierte Prinzip der conditional perfection (CP) heranzieht, nach dem Konditionalbeziehungen der Art A --> B eine Implikatur der Art A --> B auszulösen vermögen. (Siehe hierzu vor allem Geis/Zwicky 1971 und de Cornulier 1983.) Letzterer führt die Ableitung der betreffenden Implikaturen auf das Wirken des pragmatischen Prinzips der Exhaustivität der Nennung von Bedingungen zurück.)

Wenn man nämlich annimmt, daß mit der Präsupposition ${ }^{p}$--> q, die von der Partikel auch für Konditionalkonstruktionen mit der Bedeutung $\mathrm{p} \mathrm{-->} \mathrm{q} \mathrm{induziert} \mathrm{wird,} \mathrm{eine} \mathrm{ent-}$ sprechende Implikatur ausgelöst wird, so kann man für auch wenn-Konstruktionen die Implikatur p --> q annehmen, also eine Struktur, die auch als Konzessivpräsupposition zu interpretieren ist:

(AWK') Schema der Interpretation von auch wenn-Konstruktionen:

(a) Präsupposition (von auch induziert): $\sim \mathrm{p}-->\mathrm{q}$

(b) präsuppositionale Implikatur:p -- > q

(c) Bedeutung: ( $\left.\mathrm{p}-->^{-} \mathrm{q}\right) \&(\mathrm{p}->\mathrm{q})$

ausgedrückt

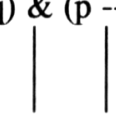

durch:

0 auch wenn

Es ist meines Erachtens diese präsuppositionale Implikatur, die in Konstruktionen mit einer Konditionalkonjunktion die Verwendung von Teilsätzen legitimiert, zwischen deren Bedeutungen erfahrungsgemäß die Beziehung $p-->$ q besteht. Diese Implikatur dürfte die Grundlage einer Erklärung dafür sein, warum Sätze wie (5)(a) im Unterschied zu solchen wie (7)(a) mit der erfahrungsgemäßen Beziehung p --> q zwischen den Bedeutungen ihrer Teilsätze informativ sind. In Sätzen wie (7)(a) wird im Unterschied zu Sätzen wie (5)(a) durch nichts formal signalisiert und dadurch legitimiert, daß aus $\mathrm{p}$ sowohl $\mathrm{q}$ als auch sein Gegenteil - q -, also Beliebiges, folgt, wodurch die entsprechende Konstruktion an sich ohne informativen Wert ist. Durch auch dagegen wird diese Ininformativität über die Implikatur, die mit der von auch induzierten Präsupposition verbunden ist, als gewollt gekennzeichnet, nämlich als Divergenz zwischen dem Inhalt einer Vorannahme und der Bedeutung der Konditionalkonstruktion.

Nun ist aber die Interpretationsstruktur von auch wenn-Konstruktionen allein aufgrund dieser Implikatur noch nicht identisch mit der von Konzessivkonstruktionen. In letzteren läßt sich die Bedeutung auf die logische Konjunktion von $\mathrm{p}$ und $\mathrm{q}$, nämlich $\mathrm{p} \& \mathrm{q}$, zurück- 
führen, und es kommt zu einem Widerspruch, wenn Präsupposition und Bedeutung in logische Konjunktion gesetzt werden. Ein solcher Widerspruch ist für auch wenn-Konstruktionen nicht ohne weiteres gegeben. Er entsteht nur, wenn der von $\mathrm{p}$ identifizierte Sachverhalt ein Faktum ist, wenn $\mathrm{p}$ wahr ist. In Kontexten, in denen dies der Fall ist, wie in dem von (5)(a), kann dann aufgrund dessen und des Bedeutungsteils p --> q mit Hilfe des Modus ponens, der hier anwendbar ist, auf die Wahrheit von q geschlossen werden. Bei Wahrheit von $p$ und $q$ kann dann die Schlußregel der Einführung der logischen Konjunktion auf $p$ und q angewandt werden, wodurch sich eine Interpretationsstruktur ergibt, die auch in Konzessivkonstruktionen wie den in (3) genannten gegeben ist, nämlich die der logischen Konjunktion p \& q.

Für die konzessive Interpretation von Verwendungen von auch wenn-Konstruktionen ergibt sich dann folgendes Schema:

(KZAWK) Schema der konzessiven Interpretation von auch wenn-Konstruktionen:

(a) Präsuppositionen:

(b) Äußerungsbedeutung:

ausgedrückt durch:
1. (von auch induziert): $\sim \mathrm{p}-->\mathrm{q}$

2. (durch Welterfahrung induziert und als Implikatur zu $\mathrm{p}-->\mathrm{q}): \mathrm{p}-->$-q

1. $(\tilde{p}->\mathrm{q}) \&(\mathrm{p}-\mathrm{p}>\mathrm{q})$

0 auch wenn

2. (vom Verwendungskontext der Konstruktion induziert): $p$

3. (nach Regeln des logischen Schließens ableitbar): p \& q

Bei der von (1) induzierten Implikatur handelt es sich gemäß den von Grice (1975) für unterschiedliche Arten von Implikaturen angeführten Kriterien und nach König (1986) und de Cornulier (1983) um eine generalisierte konversationelle Implikatur. Generalisiert deshalb, weil sie nicht vom Gebrauch eines spezifischen sprachlichen Ausdrucks abhängt, sondern vom Vorkommen einer spezifischen semantischen Einheit. Konversationell aus dem Grunde, daß sie aufgehoben werden kann. Von diesen Eigenschaften verbleibt nun der Implikatur A --> B, wenn sie von einer Präsupposition A --> B induziert wird, nur die, generalisiert zu sein. Aufhebbar ist sie in einem solchen Fall genausowenig, wie eine lexikalisch induzierte, d.h. "konventionell" induzierte logische Präsupposition aufhebbar ist (siehe hierzu Karttunen/Peters 1979:11ff.; zum Begriff der logischen Präsuppositionen siehe Pasch 1990, 1992 und 1994), und genausowenig, wie ein semantisches Implicatum aufhebbar ist. Vgl. (8):

A.: Auch wenn es dir lästig ist, mußt du mir jetzt zuhören. ?Allerdings kann niemand annehmen, daß du mir nicht zuhören mußt, wenn es dir lästig ist.

Die Implikatur ${ }^{\sim}$ A --> ${ }^{\sim}$ B ist bei den akzeptablen auch wenn- Konstruktionen schon deshalb nicht aufhebbar, weil durch die Natur der Bedeutungen der Teilsätze der Satzverknüpfung eine Welterfahrung aktiviert wird, die von der Implikatur identifiziert wird. 


\section{Fazit}

Aus dem soeben Dargelegten leite ich folgende Hypothese ab: Die auf dem Prinzip der conditional perfection beruhende Implikatur ist eine "special class of implicature" (siehe Geis/Zwicky 1971:565), eine "konzeptuelle Implikatur". Konzeptuelle Implikaturen, die von Präsuppositionen induziert werden, sind nicht in der Weise aufhebbar, wie es Implikaturen sind, die von Aspekten.der Bedeutung induziert werden. Sie können als aktuelle Inferenzen nur durch die Bedeutung verhindert werden, für die die Präsupposition induziert wird, durch die sie selbst induziert werden (siehe die konzessive Intepretation von auch wenn-Konstruktionen nach (KZAWK)). Ein möglicher Grund hierfür ist, daß die sie induzierenden Präsuppositionen zum konzeptuellen Hintergrund der Bedeutung der sprachlichen Äußerung gehören, vor dem die Bedeutung des verwendeten sprachlichen Ausdrucks erst einen Sinn ergibt.

Als Fazit für die Diskussion um das Phänomen der Implikaturen ergibt sich für mich, daß, wenn die Aufhebbarkeit einer Implikatur derart beschränkt ist, die von Grice (1975) formulierten und in der Literatur (siehe Levinson 1983:113) verfeinerten Kriterien für die Konversationalität von Implikaturen noch weiter verfeinert werden müssen.

\section{Literatur}

Cornulier, B.de (1983): "'If' and the Presumption of Exhaustivity". - Journal of Pragmatics 7, 247-249.

Geis, M.L., Zwicky, A.M. (1971): "On Invited Inferences". - Linguistic Inquiry 2, 561-566.

Givón, T. (1978): "Negation in Language: Pragmatics, Function, Ontology". - P. Cole (ed.): Syntax and Semantics. Volume 9: Pragmatics (New York etc.: Academic Press), 69-112.

Grice, H.P. (1975): "Logic and Conversation". - P. Cole, J.L. Morgan (eds.): Syntax and Semantics. Volume 3: Speech Acts. (New York etc.: Academic Press), 43-58.

Karttunen, L., Peters, S. (1979): "Conventional Implicature". - Ch.-K. Oh, D.A. Dinneen (eds.): Syntax and Semantics. Volume 11: Presupposition (New York etc.: Academic Press), 1-56.

König, E. (1986): "Conditionals, Concessive Conditionals, and Concessives: Areas of Contrast, Overlap and Neutralization". - E.C. Traugott et al. (eds.): On Conditionals. (Cambridge etc.: Cambridge University Press), 229-246.

König, E., Eisenberg, P. (1984):" Zur Pragmatik von Konzessivsätzen". - G. Stickel (Hg.): Jahrbuch 1983 des Instituts für deutsche Sprache: Pragmatik in der Grammatik. (Düsseldorf: Schwann), 313-332.

Levinson, S.C. (1983): Pragmatics. - Cambridge etc.: Cambridge University Press.

Pasch, R. (1990): "Towards a Uniform Pragmatic Description of Logical and Other Presuppositions". - W. Bahner, J. Schildt, D. Viehweger (Hgg.): Proceedings of the Fourteenth International Congress of Linguists, Berlin-GDR, August 10 - August 15, 1987 (Berlin: Akademie-Verlag), 1017-1019.

-- (1992): "Kausale, konzessive und adversative Konnektive: Konnektive als Mittel des Ausdrucks von Diskurspräsuppositionen". - MLL (Münstersches Logbuch zur Linguistik) 1: Semantik, hrsg. von S. Beckmann, 33-48.

-- (1994): Konzessivität von 'wenn'-Konstruktionen. - Tübingen: Narr (= Forschungsberichte des Instituts für deutsche Sprache 72).

Wessel, H. (1984): Logik. - Berlin: VEB Deutscher Verlag der Wissenschaften. 